\title{
Regeneration of Anti-Protons in Ultrarelativistic Heavy-Ion Collisions *
}

\author{
Ralf Rapp and Edward V. Shuryak \\ Department of Physics and Astronomy, SUNY at Stony Brook, NY 11794-3800, USA
}

The production and annihilation of antiprotons in the hadronic phase of heavy-ion collisions is evaluated within a thermal equilibrium approach. It is shown that the inverse reaction of $p \bar{p}$ annihilation (i.e., multi-pion annihilation $N_{\pi} \pi \rightarrow p \bar{p}$ with $N_{\pi} \simeq 5-7$ ) in connection with oversaturation of pion phase space (i.e., finite pion chemical potentials) plays an important role in understanding the observed antiproton yields at SpS energies within the standard picture of subsequent chemical and thermal freezeout. Implications for RHIC energies are also addressed.

\section{Introduction}

An important question in heavy-ion collisions at high energies concerns whether reinteractions between produced particles are frequent enough to establish and maintain thermal (and/or chemical) equilibrium, which constitutes an inevitable prerequisite to investigate the phase diagram of QCD. One way of addressing this issue is by testing predictions of equilibrium approaches with a large class of observables, aiming at a consistent description within one common scenario. In the context of the SpS heavy-ion experiments, this program has been carried out with remarkable success, as reflected by a simultaneous description of hadron abundances [1], $p_{t}$-spectra encoding various patterns of hydrodynamic (collective) flow effects [2], two-particle correlations [3], etc.. The deduced picture is that of a subsequent chemical and thermal freezeout, being characterized by the respective points $\left(\mu_{N}^{\text {chem }}, T_{\text {chem }}\right) \simeq(270,170) \mathrm{MeV}$ [1] and $\left(\mu_{N}^{\text {therm }}, T_{\text {therm }}\right) \simeq(410,120) \mathrm{MeV}$ in terms of temperature and nucleon chemical potential coordinates of the phase diagram. The dynamical justification resides in a hierarchy of hadronic interaction strengths: on the one hand, typical elastic cross sections are large $(\sim 100 \mathrm{mb})$, supporting thermal equilibrium between $T_{\text {chem }}$ and $T_{\text {therm }}$ for about $5-10 \mathrm{fm} / \mathrm{c}$; on the other hand, inelastic (numberchanging) reactions have cross sections of 1-2 orders of magnitude smaller so that the net abundances of stable particles (nucleons, pions, kaons) are not significantly altered during the hadronic phase. Furthermore, the nontrivial features observed in electromagnetic spectra, such as the enhancement found in both low- and intermediate-mass dilepton as well as direct photon spectra, can also be accounted for within the same thermodynamic framework (cf. ref. [4] for a recent review).

In the following we investigate an observable - antiproton yields - that so far has been difficult to accommodate in this viewpoint of the collision dynamics, cf. also ref. [5] and its application to antihyperons in ref. [6].

\footnotetext{
*work supported by US-DOE grant DE-FG02-88ER40388.
} 


\section{Antiproton Production at CERN-SpS Energies}

A copious production of antiprotons has been among early suggestions for signals of Quark-Gluon Plasma formation in heavy-ion reactions [7] (based on the much reduced threshold for anti-particle production in a partonic as compared to a hadronic environment). At the $\mathrm{SpS}$ the antiproton-to-proton ratio measured in central $\mathrm{Pb}(158 \mathrm{AGeV})-\mathrm{Pb}$ collisions [8] is in agreement with the value predicted by the corresponding hadrochemical freezeout, $\bar{p} / p=\exp \left[-2 \mu_{N}^{\text {chem }} / T_{\text {chem }}\right] \simeq 5 \%$. The large annihilation cross section of antiprotons $\left(\sigma_{p \bar{p}}^{a n n} \simeq 50 \mathrm{mb}\right.$ at the relevant thermal energies of $\left.\sqrt{s} \simeq 2.3 \mathrm{GeV}\right)$ renders this a rather puzzling fact, since the pertinent chemical relaxation time,

$\tau_{\bar{p}}^{c h e m}=\frac{1}{\sigma_{p \bar{p}}^{a n n}\left(s_{t h}\right) \varrho_{B} v_{t h}}$

stays below the fireball lifetime until rather late in the hadronic evolution, cf. fig. 1. Indeed, standard hadronic transport calculations substantially underestimate the antipro-

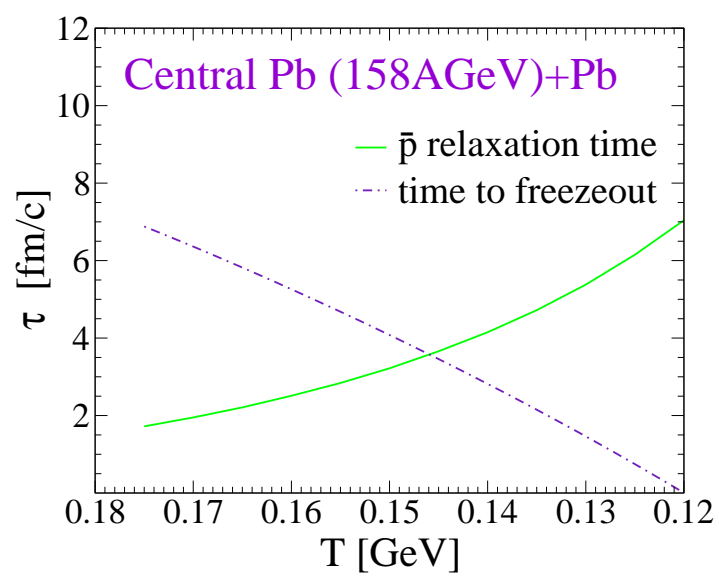

Figure 1. Chemical relaxation time for antiprotons (solid line), eq. (11), and remaining fireball lifetime until thermal freezeout (dashed-dotted line) as obtained in the thermal model of ref. [1].

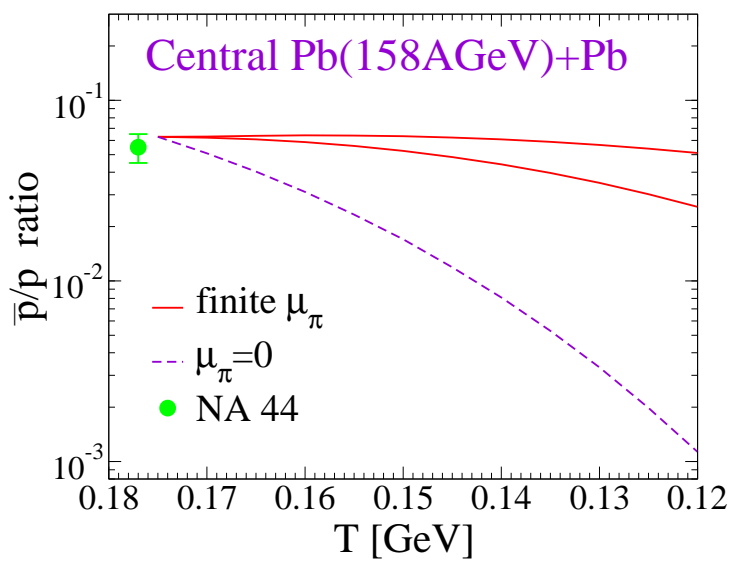

Figure 2. Temperature evolution of the $\bar{p} / p$ ratio with zero (dashed line) and finite (solid lines) pion chemical potentials. The two solid lines reflect uncertainties corresponding to $\mu_{\pi}^{\text {therm }}=65-80 \mathrm{MeV}$.

ton production. This led to speculations on various in-medium effects such as a shielding of the annihilation [9] or enhanced production via an increased string tension in the prehadronic stages [10]. However, present transport approaches are not able to consistently treat reactions with more than 2 particles in the incoming channel. Thus, although $p \bar{p}$ annihilation is included, the inverse reaction of multi-pion annihilation is not, entailing a violation of detailed balance. This observation alone, though, does not yet resolve the puzzle: a naive estimate assuming an equilibrium $\bar{p}$ abundance at thermal freezeout results in $\bar{p} / p=\exp \left[-2 \mu_{N}^{\text {therm }} / T_{\text {therm }}\right]=0.1 \%$, a factor $\sim 50$ below the measured value. What is missing here is that, after chemical freezeout - due to effective pion-number conservation - the thermal pion densities exceed their chemical equilibrium values. In statistical mechanics language this can be described by the build-up of finite pion chemical potentials. 
The thermal rate equation for $p \bar{p} \leftrightarrow N_{\pi} \pi$ then takes the form

$\mathcal{R}_{t h}=\int d^{3} \tilde{k}_{p} d^{3} \tilde{k}_{\bar{p}} d^{3} \tilde{k}_{1} \cdots d^{3} \tilde{k}_{N_{\pi}} \delta^{(4)}\left(K_{t o t}\right)\left|\mathcal{M}_{N_{\pi}}\right|^{2}\left\{z_{p} z_{\bar{p}} \mathrm{e}^{-\left(E_{p}+E_{\bar{p}}\right) / T}-z_{\pi}^{N_{\pi}} \mathrm{e}^{-\sum_{i=1}^{N_{\pi}} \omega_{i} / T}\right\}$,

where $\mathcal{M}_{N_{\pi}}$ is the invariant scattering matrix element. The 4-momentum conserving $\delta$ function forces the sum of proton and antiproton energies $E_{p(\bar{p})}$ to equal the sum of pion energies $\omega_{i}$. Insisting on chemical equilibrium for the $p \bar{p} \leftrightarrow N_{\pi} \pi$ reaction thus provides an equation for the antiproton fugacity, $z_{\bar{p}}=z_{\pi}^{N_{\pi}} z_{p}$, implying large enhancement factors in the presence of a finite $\mu_{\pi}$ (note that changes in $z_{\bar{p}}$ have negligible feedback on $z_{\pi}$ or $z_{p}$ under SpS conditions). A more detailed calculation [5] including measured pionmultiplicity distributions and $c m s$-energy dependencies in $p \bar{p}$ annihilation [12] yields an average fugacity factor

$\left\langle z_{\pi}^{N_{\pi}}\right\rangle=\sum_{N_{\pi}=2}^{N_{\pi}^{\max }} w_{N_{\pi}} \exp \left[N_{\pi} \mu_{\pi} / T\right]$

with $w_{N_{\pi}}$ the probability weight of the $N_{\pi}$-pion channel. The temperature dependence of the ratio $\bar{p} / p=\left\langle z_{\pi}^{N_{\pi}}(T)\right\rangle \exp \left[-2 \mu_{N}^{\text {chem }}(T) / T_{\text {chem }}\right]$ in relative chemical equilibrium is displayed in fig. 2 based on the thermal fireball model of ref. [11]. As a result of large enhancement factors, the antiproton abundance at $T_{\text {chem }}$ can essentially be supported towards thermal freezeout (where $\left\langle z_{\pi}^{N_{\pi}}\right\rangle \simeq 25-50$ ), thus demonstrating the importance of multi-pion back-reactions in the presence of pion oversaturation.

\section{Antiproton Production at RHIC Energies}

The same approach can be applied to higher collision energies. First data from RHIC [13] have shown a $\bar{p} / p$ ratio of $\sim 60 \%$ at $\sqrt{s}=130 \mathrm{AGeV}$, implying $\mu_{N}^{\text {chem }} \simeq 45 \mathrm{MeV}$ (assuming $T_{\text {chem }}=180 \mathrm{MeV}$ ), cf. fig. 通. Extrapolating to $\sqrt{s}=200 \mathrm{AGeV}$ yields $\mu_{N}^{\text {chem }}=27 \mathrm{MeV}$

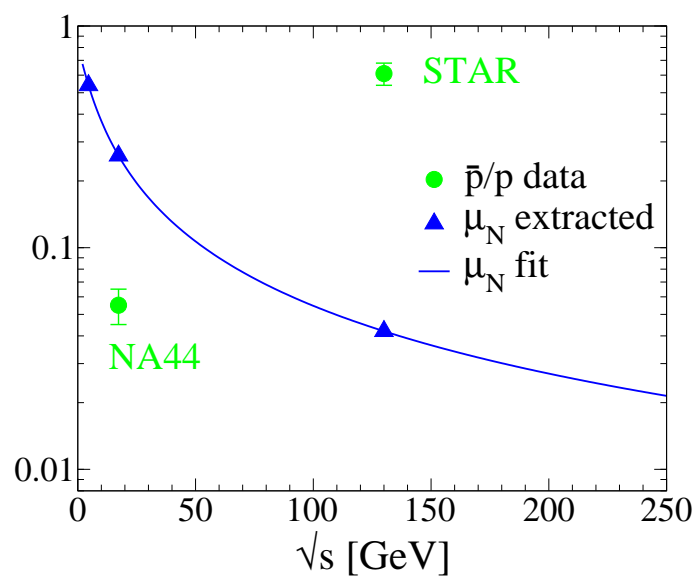

Figure 3. Excitation function of the nucleon chemical potential (in $[\mathrm{GeV}]$ ) at chemical freezeout, being constrained by measured $\bar{p} / p$ ratios [8,13] (filled dots).

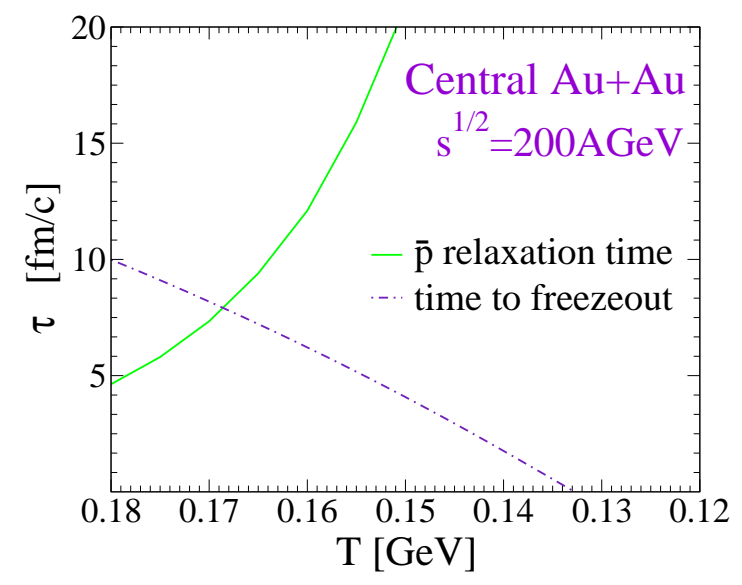

Figure 4. Chemical relaxation time for antiprotons (solid line) in comparison to the remaining fireball lifetime in a thermal model at full RHIC energy [14]. 
corresponding to $\bar{p} / p=75 \%$. Contrary to $\mathrm{SpS}$ conditions, the chemical relaxation times for antiprotons quickly exceed the fireball lifetime (cf. fig. (1), i.e., multi-pion annihilation is not frequent enough to support the comparatively large antiproton abundances. Thus, the $\bar{p} / p$ ratio at full RHIC energy should be more directly associated with the chemical freezeout stage (the reduction in net-baryon number also leads to a much less pronounced pion oversaturation towards thermal freezeout [14]).

\section{Conclusions}

We have shown that the backward reaction in $p \bar{p} \leftrightarrow N_{\pi} \pi$ (with $N_{\pi} \simeq 6$ ) gives important contributions to antiproton production in the hadronic phase of heavy-ion collisions at CERN-SpS energies. Coupled with realistic estimates of pion oversaturation effects, one finds a $\bar{p} / p$ ratio that with decreasing temperature deviates little from its value at chemical freezeout, sustaining agreement with experiment. This resolves the naive expectation of large annihilation losses towards thermal freezeout, and lends further support to an equilibrium picture of central $\mathrm{Pb}-\mathrm{Pb}$ collisions. Medium effects in $p \bar{p} \leftrightarrow N_{\pi} \pi$ reactions are neither required nor excluded, but might be addressed within a time-dependent treatment of the rate equations.

\section{ACKNOWLEDGEMENT}

We thank K. Redlich for interesting discussion.

\section{REFERENCES}

1. P. Braun-Munzinger, I. Heppe and J. Stachel, Phys. Lett. B465 (1999) 15.

2. C.M. Hung and E.V. Shuryak, Phys. Rev. C57 (1998) 1891.

3. U.A. Wiedemann and U. Heinz, Phys. Rep. 319 (1999) 145.

4. R. Rapp and J. Wambach, hep-ph/9909229 and Adv. Nucl. Phys. 25 (2000) 1.

5. R. Rapp and E.V. Shuryak, hep-ph/0008326 and Phys. Rev. Lett. 86 (2001) 2980.

6. C. Greiner and S. Leupold, nucl-th/0009036; C. Greiner, these proceedings.

7. U. Heinz, P.R. Subramanian, H. Stöcker and W. Greiner, J. Phys. G12 (1986) 1237;

P. Koch, B. Müller, H. Stöcker and W. Greiner, Mod. Phys. Lett. A3 (1998) 737.

8. M. Kaneta for the NA44 collaboration, Nucl. Phys. A638 (1998) 419c; G.I. Veres for the NA49 collaboration, Nucl. Phys. A661 (1999) 383c.

9. Y. Pang, D.E. Kahana, S.H. Kahana and H. Crawford, Phys. Rev. Lett. 78 (1997) 3418.

10. M. Bleicher et al., Phys. Lett. B485 (2000) 133.

11. R. Rapp and J. Wambach, Eur. Phys. J. A6 (1999) 415.

12. C.B. Dover, T. Gutsche, M. Maruyama and A. Faessler, Prog. Part. Nucl. Phys. 29 (1992) 87.

13. J.W. Harris for the STAR collaboration, these proceedings;

W.A. Zajc for the PHENIX collaboration, these proceedings.

14. R. Rapp, hep-ph/0010101 and Phys. Rev. C (2001) in print. 